\title{
CT image visual quantitative evaluation and clinical classification of coronavirus disease (COVID-19)
}

\author{
Kunwei $\mathrm{Li}^{1,2} \cdot$ Yijie Fang ${ }^{1,2} \cdot$ Wenjuan $\mathrm{Li}^{1,2} \cdot$ Cunxue Pan ${ }^{1,2} \cdot$ Peixin Qin ${ }^{1,2} \cdot$ Yinghua Zhong $^{1,2} \cdot$ Xueguo Liu ${ }^{1,2}$. \\ Mingqian Huang ${ }^{3} \cdot$ Yuting $\mathrm{LiaO}^{4} \cdot$ Shaolin $\mathrm{Li}^{1,2}$
}

Received: 18 February 2020 / Revised: 13 March 2020 / Accepted: 16 March 2020 / Published online: 25 March 2020

(C) European Society of Radiology 2020

\begin{abstract}
Objectives To explore the relationship between the imaging manifestations and clinical classification of COVID-19.

Methods We conducted a retrospective single-center study on patients with COVID-19 from Jan. 18, 2020 to Feb. 7, 2020 in Zhuhai, China. Patients were divided into 3 types based on Chinese guideline: mild (patients with minimal symptoms and negative CT findings), common, and severe-critical (patients with positive CT findings and different extent of clinical manifestations). CT visual quantitative evaluation was based on summing up the acute lung inflammatory lesions involving each lobe, which was scored as $0(0 \%), 1(1-25 \%), 2(26-50 \%), 3(51-75 \%)$, or $4(76-100 \%)$, respectively. The total severity score (TSS) was reached by summing the five lobe scores. The consistency of two observers was evaluated. The TSS was compared with the clinical classification. ROC was used to test the diagnosis ability of TSS for severe-critical type.

Results This study included 78 patients, 38 males and 40 females. There were 24 mild (30.8\%), 46 common (59.0\%), and 8 severecritical $(10.2 \%)$ cases, respectively. The median TSS of severe-critical-type group was significantly higher than common type $(p<0.001)$. The ICC value of the two observers was 0.976 (95\% CI 0.962-0.985). ROC analysis showed the area under the curve (AUC) of TSS for diagnosing severe-critical type was 0.918 . The TSS cutoff of 7.5 had $82.6 \%$ sensitivity and $100 \%$ specificity. Conclusions The proportion of clinical mild-type patients with COVID-19 was relatively high; CT was not suitable for independent screening tool. The CT visual quantitative analysis has high consistency and can reflect the clinical classification of COVID-19.

Key Points

- CT visual quantitative evaluation has high consistency (ICC value of 0.976) among the observers. The median TSS of severecritical type group was significantly higher than common type $(p<0.001)$.

- ROC analysis showed the area under the curve (AUC) of TSS for diagnosing severe-critical type was 0.918 (95\% CI $0.843-$ 0.994). The TSS cutoff of 7.5 had $82.6 \%$ sensitivity and $100 \%$ specificity.

- The proportion of confirmed COVID-19 patients with normal chest CT was relatively high (30.8\%); CT was not a suitable screening modality
\end{abstract}

Keywords COVID-19 · Tomography, X-ray computed $\cdot$ Quantitative evaluation $\cdot$ Classification

Kunwei Li and Yijie Fang contributed equally to this work.

\section{Shaolin Li}

lishaolin1963@126.com

1 Radiology Department, The Fifth Affiliated Hospital of Sun Yat-sen University, 52 East Meihua Road, New Xiangzhou,

Zhuhai 519000, Guangdong Province, China
2 Guangdong Provincial Key Laboratory of Biomedical Imaging, The Fifth Affiliated Hospital of Sun Yat-sen University, 52 East Meihua Road, New Xiangzhou, Zhuhai 519000, Guangdong Province, China

3 Department of Diagnostic, Molecular and Interventional Radiology, Icahn School of Medicine at Mount Sinai , 1 Gustave Levy Place, New York, NY 10029, USA

4 GE Healthcare, Guangzhou 510623, China 


$\begin{array}{ll}\text { Abbreviations } \\ \text { AUC } & \begin{array}{l}\text { Area under the curve } \\ \text { COVID }\end{array} \\ \text { Coronavirus disease } \\ \text { ICC } & \begin{array}{l}\text { Intragroup correlation coefficient } \\ \text { Receiver operating characteristic }\end{array} \\ \text { ROC } & \begin{array}{l}\text { Reverse-transcriptase } \\ \text { polymerase-chain-reaction }\end{array} \\ \text { RT-PCR } & \text { Severe acute respiratory syndrome } \\ \text { SARS-CoV } & \text { conavirus } \\ \text { TSS } & \text { Total severity score }\end{array}$

\section{Introduction}

Since December 2019, a number of cases of pneumonia with fever, cough, and dyspnea as clinical manifestations have been found in Wuhan, Hubei Province, China [1]. The analysis of the whole genome sequence of the respiratory samples suggests that it is a new type of betacoronavirus [2], which resembled severe acute respiratory syndrome coronavirus (SARSCoV) [3]. On February 11, 2020, the World Health Organization (WHO) officially named it coronavirus disease (COVID-19).

WHO has recently declared the outbreak a public health emergency of international concern [4]. As of March 12, 2020, 124,922 laboratory-confirmed and clinical-confirmed cases have been documented globally (i.e., the USA, Vietnam, Germany) [4-7], 80,980 laboratory-confirmed and clinical-confirmed cases and 3173 deaths in China as of March 12, 2020 [8]. On Jan. 15, 2020, the first confirmed family cluster was reported in Zhuhai, China, where the parents presented with unexplained pneumonia after coming from Wuhan to visit their daughter who was living in Zhuhai, China; afterwards, the daughter also developed respiratory symptoms and infection with SARS-CoV-2 was confirmed.

As of February 13, the journal Radiology has published several articles on the imaging features of COVID-19 [9-12], but all of them are descriptive analyses. In February 2020, the Chinese Society of Radiology issued the radiologic diagnosis of pneumonia with COVID-19. CT plays an important role in the screening and diagnosis of COVID-19. The first edition of the experts [13] divided CT manifestations into three stages: early, progressive, and severe according to the extent and features of the pulmonary abnormalities. However, it did not clarify the relationship between the extent of inflammation and the clinical presentation of the patient. In this study, we used a simple convenient method to quantify the imaging findings.

\section{Methods}

\section{Case selection}

We performed a retrospective, single-center study of the SARS-CoV-2 laboratory-confirmed cases with which included 78 cases between Jan. 18, 2020 and Feb. 7, 2020 in Zhuhai, China. A confirmed case was defined as positive by highthroughput sequencing or real-time reverse-transcriptase polymerase-chain-reaction (rRT-PCR) assay of nasal and pharyngeal swab specimens [1]. The rRT-PCR test kits used on the patients in this study was manufactured by Shanghai Zhijiang Biotechnology Co. This study was approved by the ethics committee of the Fifth Affiliated Hospital of Sun Yatsen University and the requirement for informed consent was waived since the study had no risk and would not adversely affect the subjects' rights or welfare. Patient selection for this study was consecutive, and no exclusion criteria were applied.

\section{CT image acquisition}

All scans were performed with the patient in the supine position during end-inspiration without intravenous contrast on two CT scanners, uCT 760 and uMI 780 scanners (United Imaging). The scanning range was from the apex to lung base. All images were obtained with a standard dose scanning protocol, reconstructed at $1.0 \mathrm{~mm}$ slice thickness, with $1 \mathrm{~mm}$ increment, $512 \mathrm{~mm} \times 512 \mathrm{~mm}$, and a sharp reconstruction kernel (B_VSHARP_B). Lung window setting was with a window level of -600 Hounsfield units (HU) and window width of $1500 \mathrm{HU}$.

\section{Image interpretation}

Image analysis was performed using the institutional digital database system (Neusoft V5.5.4.50720). All CT images were reviewed by two radiologists with 5 and 3 years of experience in imaging (Y.F. and W.L.). Imaging was reviewed independently and final decisions reached by consensus. For disagreement between the two primary radiologist interpretations, a third experienced thoracic radiologist with 17 years of experience (K.L.) adjudicated a final decision. No negative control cases were examined.

For each of the 78 patients, the CT scan was evaluated for the following characteristics: (1) distribution: presence of peripheral or peribronchovascular; (2) density: presence of ground-glass opacities, mixed ground-glass opacities, or consolidation; (3) internal structures: presence of air bronchogram, interlobular septal thickening, cavitation; (4) number of lobes affected by ground-glass or consolidative opacities; (5) presence of fibrotic lesions; (6) presence of centrilobular nodules; (7) presence of a pleural effusion; (8) presence of thoracic lymphadenopathy (defined as lymph 
node size of $\geq 10 \mathrm{~mm}$ in short-axis dimension); and (9) presence of underlying lung disease such as tuberculosis, emphysema, or interstitial lung disease were noted. Ground-glass opacification was defined as hazy increased lung attenuation with preservation of bronchial and vascular margins and consolidation was defined as opacification with obscuration of margins of vessels and airway walls [14].

\section{CT visual quantitative evaluation}

Two radiologists (Y.F. and W.L.) reviewed all images independently blinded to the clinical information. Percentage of involvement in each lobe was recorded as well as the overall lung "total severity score (TSS)". Each of the five lung lobes was assessed for percentage of the lobar involvement and classified as none (0\%), minimal (1-25\%), mild (26-50\%), moderate (51-75\%), or severe (76-100\%), with corresponded score as $0,1,2,3$, or 4 . The TSS was reached by summing the five lobe scores (range from 0 to 20) [9]. The final score of each case was decided by a third experienced thoracic radiologist (K.L.).

\section{Clinical classifications}

All cases were divided into four groups: minimal, common, severe, and critical according to whether there were clinical symptoms, severity of pneumonia, respiratory failure, shock, other organ failure, etc., based on the Diagnosis and Treatment Plan of COVID-19 issued by National Health Commission (7th ed.) (in Chinese) [15]. (1) Mild type: mild clinical symptoms without pneumonia in imaging; (2) common type: fever, respiratory tract and other symptoms with pneumonia in imaging; (3) severe type: respiratory distress, respiratory rate $\geq 30$ times $/ \mathrm{min}$; in resting state, oxygen saturation $\leq 93 \% ; \mathrm{PaO} 2 / \mathrm{FiO} 2$ $\leq 300 \mathrm{MMHG}$; (4) critical type: respiratory failure requiring mechanical ventilation, shock and other organ failure requiring ICU monitoring and treatment.

\section{Statistical analysis}

Statistical analysis was performed using IBM SPSS Statistics for Windows, Version 25.0 (IBM Corp.). Continuous data conforming to normal distribution expressed by mean \pm standard deviation; for those not conformed (median, P25, p75) were listed. Intragroup correlation coefficient (ICC) was used to test the consistency of TSS scores of two observers, ICC values $<0.4$, $0.4 \sim 0.75$, and $>0.75$ represent poor, moderate, and good repeatability, respectively. The distribution balance of involved lobes and the number of involved lobes in different clinical types were compared by chi-squared test or Fisher exact test when sample sizes were small and by analysis of variance tests. Wilcoxon-rank test was used for comparison of TSS among different clinical types, since TSS did not conform to the normal distribution. ROC was used to test the differential diagnosis ability of TSS in common-type group and severe-critical-type group.

\section{Results}

\section{Demographic and clinical characteristics}

Seventy-eight patients were included in the study. The clinical subtype classification was as follows: 24 (30.8\%) had minimal, 46 (59.0\%) had common, 6 (7.7\%) had severe, and 2 (2.6\%) had critical disease. The demographic data for all patients are shown in Table 1. All patients were discharged after a mean hospitalized period of $20 \pm 7$ days (range 9-45 days). No patients died in this cohort.

\section{Inter-observer consistency of CT visual quantitative evaluation}

The consistency test results of CT visual quantitative analysis of two observers showed good repeatability with ICC 0.976 (95\% confidence interval 0.962-0.985).

\section{Imaging findings}

Of 78 patients who underwent chest computed tomography on admission, $71.8 \%$ (56/78) had CT evidence of pneumonia. Among 56 pneumonia patients, 45 cases $(80.4 \%)$ had ground-glass opacities, $43(76.8 \%)$ had mixed ground-glass opacities, $12(21.4 \%)$ had consolidation, $49(87.5 \%)$ had peripheral distribution, 18 (32.1\%) had peribronchovascular distribution, $25(44.6 \%)$ had interlobular septal thickening within the lesions, 41 (73.2\%) had air bronchograms, $30(53.6 \%)$ had fibrotic lesions, no cavitation was seen, and $5(8.9 \%)$ had pleural effusion. No centrilobular nodules or lymphadenopathy was found.

Pneumonias were in both lower lobes in $48(85.7 \%)$ cases, in the left upper lobe in 42 (75.0\%), right upper lobe in 32 $(57.1 \%)$, and right middle lobe in $30(53.6 \%)$. Eight cases involved a single lung lobe, accounting for $10.3 \%$ of all cases and for $14.8 \%$ of CT positive cases; 40 cases involved more than two lung lobes, accounting for $51.3 \%$ of all cases and for $74 \%$ of CT positive cases; 45 cases involved both lungs, accounting for $57.7 \%$ of all cases and for $83.3 \%$ of CT positive cases. 
Table 1 Characteristics of the patient cohort

\begin{tabular}{|c|c|c|}
\hline & Characteristics & All patients $(n=78)$ \\
\hline \multirow[t]{2}{*}{ Sex } & Male & $38(48.7 \%)$ \\
\hline & Female & $40(51.3 \%)$ \\
\hline Age & & $44.6 \pm 17.9$ \\
\hline \multirow[t]{3}{*}{ Epidemiological history } & Recent travel to Hubei & $60(76.9 \%)$ \\
\hline & Exposure to infected people & $13(16.7 \%)$ \\
\hline & Unknown exposure & $5(6.4 \%)$ \\
\hline \multirow[t]{8}{*}{ Basic diseases } & Hypertension & $10(12.8 \%)$ \\
\hline & Diabetes & $4(5.1 \%)$ \\
\hline & Chronic liver disease & $1(1.3 \%)$ \\
\hline & Chronic obstructive pulmonary disease & $9(11.5 \%)$ \\
\hline & Heart disease & $2(2.6 \%)$ \\
\hline & Tumor & $3(3.8 \%)$ \\
\hline & Other types of metabolic diseases & $3(3.8 \%)$ \\
\hline & Cerebrovascular disease & $1(1.3 \%)$ \\
\hline \multirow[t]{3}{*}{ Smoking history } & Never smoker & $71(91.0 \%)$ \\
\hline & Current smoker & $5(6.4 \%)$ \\
\hline & Former smoker & $2(2.6 \%)$ \\
\hline \multirow[t]{21}{*}{ Clinical symptoms } & Normal & $24(30.8 \%)$ \\
\hline & Fever & $54(69.2 \%)$ \\
\hline & $37.3-38^{\circ} \mathrm{C}$ & $32(41.0 \%)$ \\
\hline & $38.1-39^{\circ} \mathrm{C}$ & $20(25.6 \%)$ \\
\hline & $>39^{\circ} \mathrm{C}$ & $2(2.6 \%)$ \\
\hline & Asymptomatic & $7(9.0 \%)$ \\
\hline & Chills & $5(6.4 \%)$ \\
\hline & Cough & $36(46.2 \%)$ \\
\hline & Sputum & $16(20.5 \%)$ \\
\hline & Hemoptysis & $2(2.6 \%)$ \\
\hline & Sore throat & $8(10.3 \%)$ \\
\hline & Nasal congestion and runny nose & $10(12.8 \%)$ \\
\hline & Headache and dizziness & $6(7.7 \%)$ \\
\hline & Chest tightness and shortness of breath & $4(5.1 \%)$ \\
\hline & Dyspnea & $1(1.3 \%)$ \\
\hline & Weakness & $8(10.3 \%)$ \\
\hline & Muscle soreness & $9(11.5 \%)$ \\
\hline & Abdominal pain & $0(0 \%)$ \\
\hline & Diarrhea & $2(2.6 \%)$ \\
\hline & Appetite & $2(2.6 \%)$ \\
\hline & Nausea and vomiting & $2(2.6 \%)$ \\
\hline \multirow[t]{4}{*}{ Onset to admission } & Median & 3 days \\
\hline & $P_{25}$ & 1 day \\
\hline & $P_{75}$ & 5 days \\
\hline & Range & $0-15$ days \\
\hline
\end{tabular}

\section{Comparison of CT visual quantitative evaluation and clinical classification}

The distribution of pulmonary lobe involvement in different clinical types is shown in Table 2. All 5 lobes were involved in the severe-critical type while the lower lobes were usually involved in the common type (40/46, 87.0\%). Compared with the severe-critical type, the common type had a lower incidence of right upper lobe and middle lobe involvement $(p=0.016 ; p=0.006$, respectively), and also a lower incidence of right lower lobe, left lower lobe, and left upper lobe involvement; however, there was no significant difference 
Table 2 Comparison of affected lobe distribution and clinical classification

\begin{tabular}{lllllll}
\hline $\begin{array}{l}\text { Frequency of lobe } \\
\text { involvement }\end{array}$ & Total (78) & $\begin{array}{l}\text { Light type } \\
(24)\end{array}$ & $\begin{array}{l}\text { Common } \\
\text { type (46) }\end{array}$ & $\begin{array}{l}\text { Severe-critical } \\
\text { type (8) }\end{array}$ & $\begin{array}{l}\text { Statistic } \\
\text { value* }\end{array}$ \\
\hline Right upper lobe & $32(41.0 \%)$ & $0(0 \%)$ & $24(52.2 \%)$ & $8(100 \%)$ & - & $0.016^{\mathrm{a}}$ \\
Right middle lobe & $30(38.5 \%)$ & $0(0 \%)$ & $22(47.8 \%)$ & $8(100 \%)$ & - & $0.006^{\mathrm{a}}$ \\
Right lower lobe & $48(61.5 \%)$ & $0(0 \%)$ & $40(87.0 \%)$ & $8(100 \%)$ & 0.225 & $0.635^{\mathrm{b}}$ \\
Left upper lobe & $42(53.8 \%)$ & $0(0 \%)$ & $34(73.9 \%)$ & $8(100 \%)$ & 1.386 & $0.239^{\mathrm{b}}$ \\
Left lower lobe & $48(61.5 \%)$ & $0(0 \%)$ & $40(87.0 \%)$ & $8(100 \%)$ & 0.225 & $0.635^{\mathrm{b}}$ \\
\hline
\end{tabular}

* $p$ value: common type vs severe-critical type

${ }^{a}$ Fisher exact test

${ }^{\mathrm{b}}$ Continuity correction between severe-critical type and common type ( $p=0.635$; $p=0.635 ; p=0.239$; respectively).

The number of involved lobes of different clinical types is shown in Table 3. Common type can involve one, two, three, and four lobes. However, due to less number of cases, there was no significant difference in the first three groups statistically. Common type and severe-critical type can both involve 5 lobes, but severe-critical type had a higher incidence than common type $(p=0.001)$. For the common type, the involved lobe number of 5 was significantly higher than 1-4 $(p=0.015)$.

The results of TSS are shown in Fig. 1. Score of mild type was 0 , while common type was 1-11 (median 5, P25 2.75, P75 6.25) and severe-critical type was 8-18 (median 10, P25 9 , P75 15.25). The score of severe-critical type was significantly higher than common type $(p<0.001)$. Figures 2 and 3 were from common-type and severe-critical-type patients, respectively.

\section{Evaluation of diagnostic ability of TSS}

ROC analysis showed the area under the curve (AUC) of TSS for diagnosing severe-critical type was 0.918 (95\%CI $0.843-$
0.994). The TSS cutoff of 7.5 had $82.6 \%$ sensitivity and $100 \%$ specificity (Fig. 4).

\section{Discussions}

COVID-19 is a new disease which is caused by betacoronavirus. The diameter of the virus particle is very small, about 60 140 nm; therefore, it is easy to reach the lung terminal structure, such as alveolar septum, alveolar wall, and interlobular septum, which causes extensive edema and lymphocyte infiltration in the lung interstitium; early alveolar exudation is not prominent, but the disease progresses rapidly [16].

\section{Imaging features}

In this study, the imaging features were consistent with the previous literature reports [9-13, 16-18] of viral pneumonia; most of the patients had ground-glass opacities and mixed ground-glass opacities; no patients demonstrated consolidation without ground-glass opacification. Subpleural distribution was common. It also occurred around the bronchovascular bundle. Air bronchograms and interlobular septal thickening were often present. No patients had
Table 3 Comparison of the number of affected lung lobes with clinical classification

\begin{tabular}{|c|c|c|c|c|c|c|}
\hline $\begin{array}{l}\text { Number of lobes } \\
\text { affected }\end{array}$ & Total (78) & $\begin{array}{l}\text { Light type } \\
\text { (24) }\end{array}$ & $\begin{array}{l}\text { Common type } \\
\text { (46) }\end{array}$ & $\begin{array}{l}\text { Severe-critical } \\
\text { type (8) }\end{array}$ & Statistic & $\begin{array}{l}p \\
\text { value* }\end{array}$ \\
\hline 0 & $24(100 \%)$ & $24(100)$ & $0(0)$ & $0(0 \%)$ & - & - \\
\hline 1 & $8(10.3 \%)$ & 0 & $8(17.4 \%)$ & $0(0 \%)$ & 0.546 & $0.460^{\mathrm{b}}$ \\
\hline 2 & $6(7.7 \%)$ & 0 & $6(13.0 \%)$ & $0(0 \%)$ & 0.225 & $0.635^{\mathrm{b}}$ \\
\hline 3 & $5(6.4 \%)$ & 0 & $5(10.9 \%)$ & $0(0 \%)$ & - & $1.000^{\mathrm{a}}$ \\
\hline 4 & $10(12.8 \%)$ & 0 & $10(21.7 \%)$ & $0(0 \%)$ & 0.937 & $0.333^{\mathrm{b}}$ \\
\hline 5 & $25(32.1 \%)$ & 0 & $17(37.0 \%)$ & $8(100 \%)$ & - & $0.001^{\mathrm{a}}$ \\
\hline $\begin{array}{l}\text { More than two lung } \\
\text { lobes }\end{array}$ & $40(51.3 \%)$ & 0 & $32(70.0 \%)$ & $8(100 \%)$ & 1.893 & $0.169^{\mathrm{b}}$ \\
\hline Bilateral lungs & $45(57.7 \%)$ & 0 & $37(80.4 \%)$ & $8(100 \%)$ & 0.734 & $0.392^{\mathrm{b}}$ \\
\hline
\end{tabular}


Fig. 1 The total severity score (TSS) of different clinical classifications. There were 24 cases of light type (31\%), 46 cases of common type $(60 \%)$, and 8 cases of severe-critical type (9\%). The median TSS was 10 in severecritical-type group (range 8-18), which was significantly higher than that of common type (median 5, ranged 1-11)

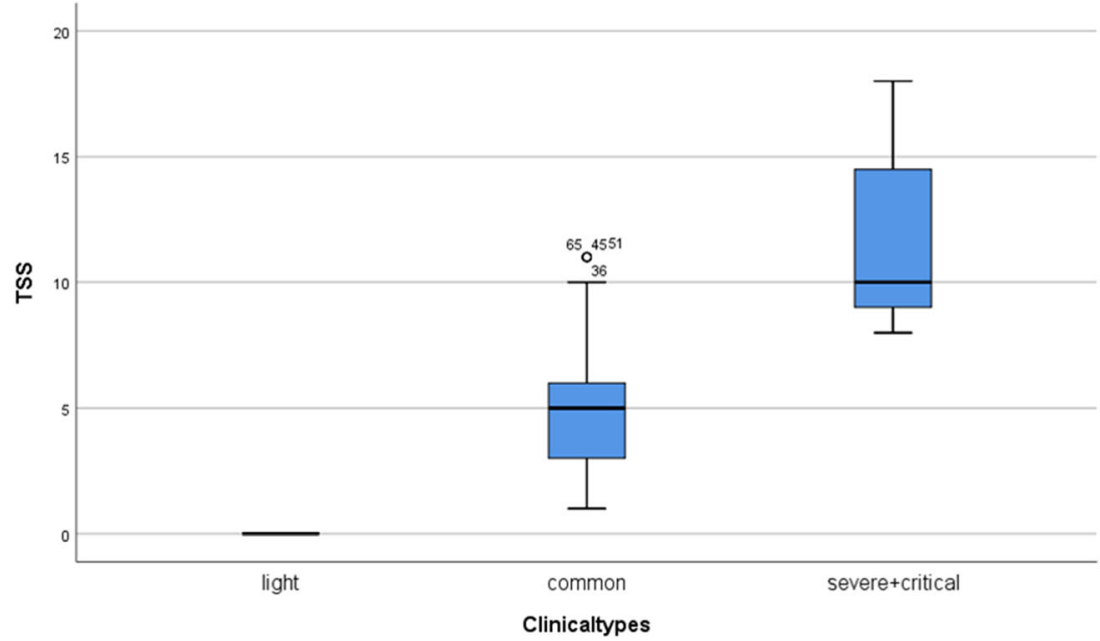

cavitation, centrilobular nodules, and lymphadenopathy. Pleural effusion was rare and most of them occurred in severe cases. Most of the lesions involved both lower lobes (85.7\%), most of them more than two lobes (74\%), and bilateral involvement was common (83.3\%); single lobe involvement was rare $(10.3 \%)$.

\section{CT visual quantitative evaluation}

In a recent study done by Michael et al, they introduced a method to score the severity of inflammation on CT images based on summing up degree of acute lung inflammation lesions involvement of each lobe (including
Fig. 2 A 32-year-old female had fever, cough, and sputum with a body temperature of $38.8^{\circ} \mathrm{C}$ for 5 days and admitted to the hospital on Jan. 27, 2020. The leukocytes and lymphocytes were decreased. She was living in Zhuhai and traveled to Wuhan on Jan. 21 and stayed there for 2 days. She was healthy and nonsmoker. Chest CT (images a-c) on the 1 st day after admission demonstrated bilateral peripheral ground-glass opacities with linear opacities. TSS was 5 . The clinical type was common type. Followup CT (images d, e) on the 20th day after onset showed peripheral shrinking consolidation with ground-grass opacities in both lungs
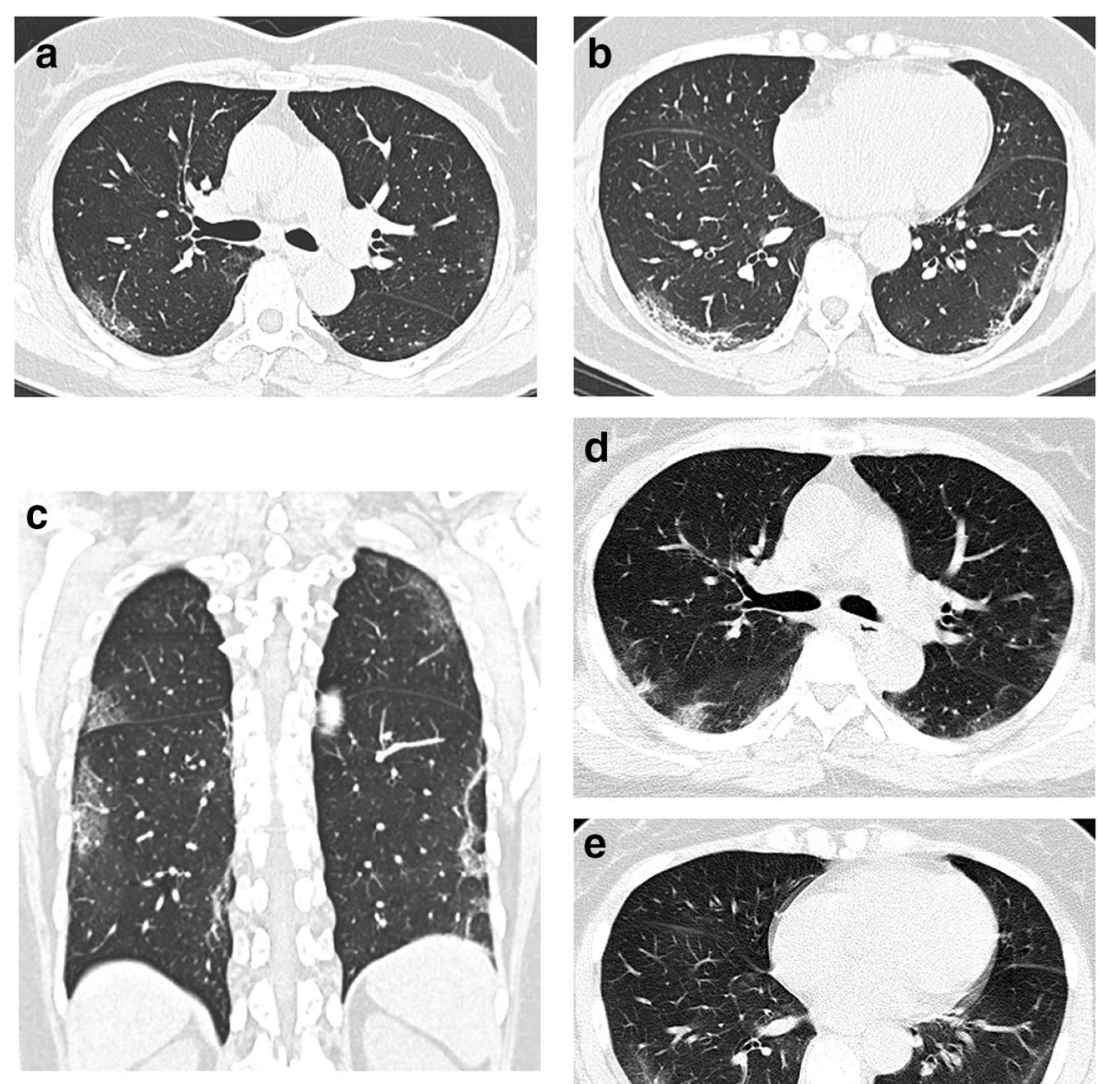

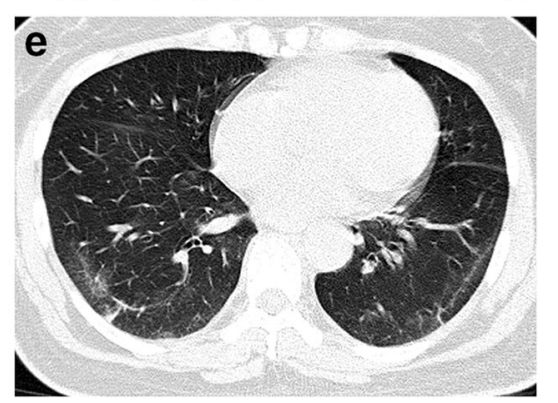


Fig. 3 A 60-year-old male was admitted to the hospital 5 days after fever and cough with a body temperature of $38^{\circ} \mathrm{C}$. The leukocytes were normal and lymphocytes were decreased. He was living in Wuhan and traveled to Zhuhai for the Spring Festival 5 days before the onset of the disease. He had tuberculosis. Chest CT (images a-c) on the 2nd day after admission demonstrated bilateral peripheral ground-glass opacities with minimal consolidation. TSS was 17. The clinical type was severe-critical type. Follow-up CT (images d, e) on the 32 nd day after onset showed bilateral fibrotic changes with ground-grass opacities with a left shift of mediastinum
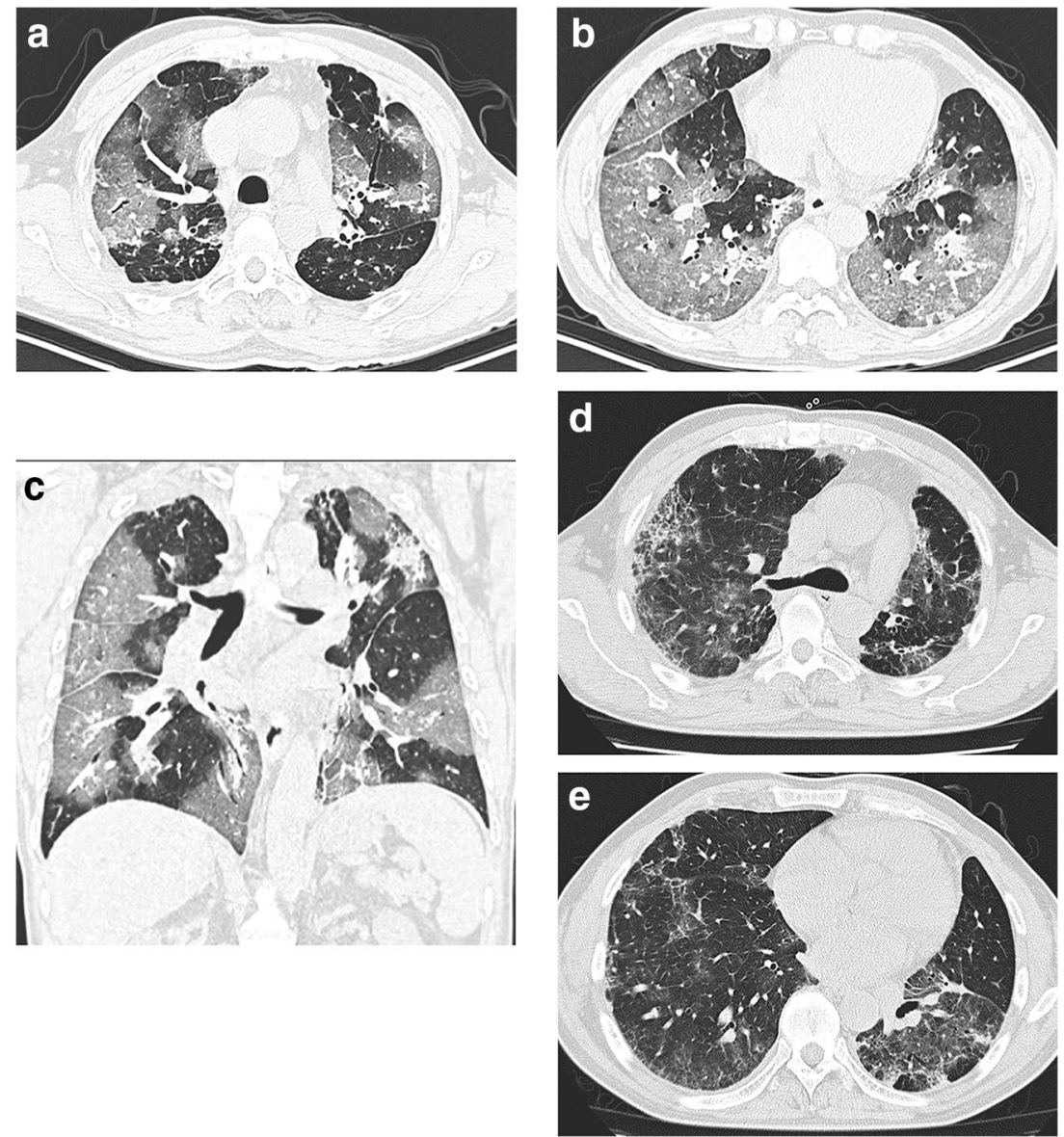

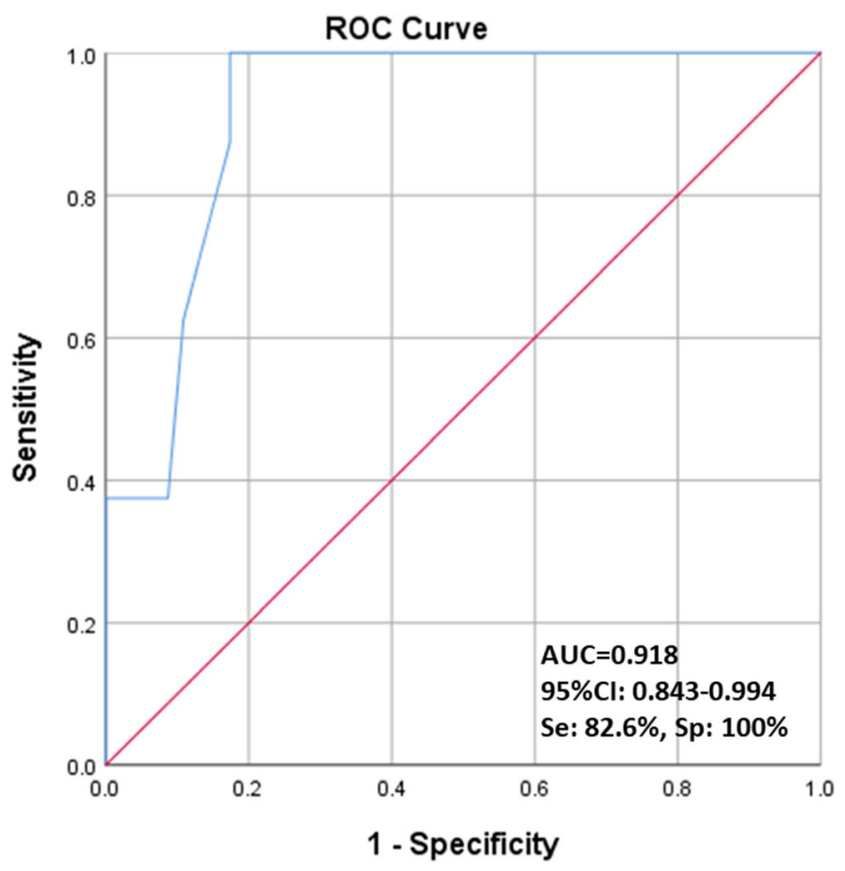

Fig. 4 TSS for diagnosing severe-critical COVID-19. Using ROC to test the differential diagnosis ability of TSS in common-type group and severe-critical-type group. ROC analysis showed the area under the curve (AUC) of TSS for diagnosing severe-critical type was $0.918(95 \% \mathrm{CI}$ $0.843-0.994)$. The TSS cutoff of 7.5 had $82.6 \%$ sensitivity and $100 \%$ specificity ground-glass opacity or consolidation or other fuzzy interstitial opacities) [9]. We used the same method to quantify pulmonary inflammation and correlate to the clinical classifications. There was significant difference in scores between common type and severe-critical type $(p<0.001)$. However, there was also a score overlap between the two groups, which showed that 8 cases in the common type had a higher score, and 5 cases in the severe-critical type had a lower score. Among 8 cases of common type, 7 cases had fibrotic lesions which indicated that the lesions began to be repaired, and all of the 8 patients were less than 70 years old (range 36-65, average 52.5 years), none of them had pulmonary complications. The specific situation of 5 patients in a severe-critical type which had a lower score was as follows: 3 of the 5 patients were over 70 years old; among them, 1 patient was a female smoker with diabetes, aged 80 years old, with moderate emphysema and a small amount of pleural effusion; 1 patient was a 70 -year-old female, with emphysema and a small amount of pleural effusion as well; the other one was 75-year-old female with high blood pressure; the forth case was a 44-year-old male without any underlying disease; however, CT images showed only progressive lesions such as ground-glass opacification and consolidation without any fibrotic lesions (Fig. 5). The last case was a 58year-old female without any underlying disease; further analysis 

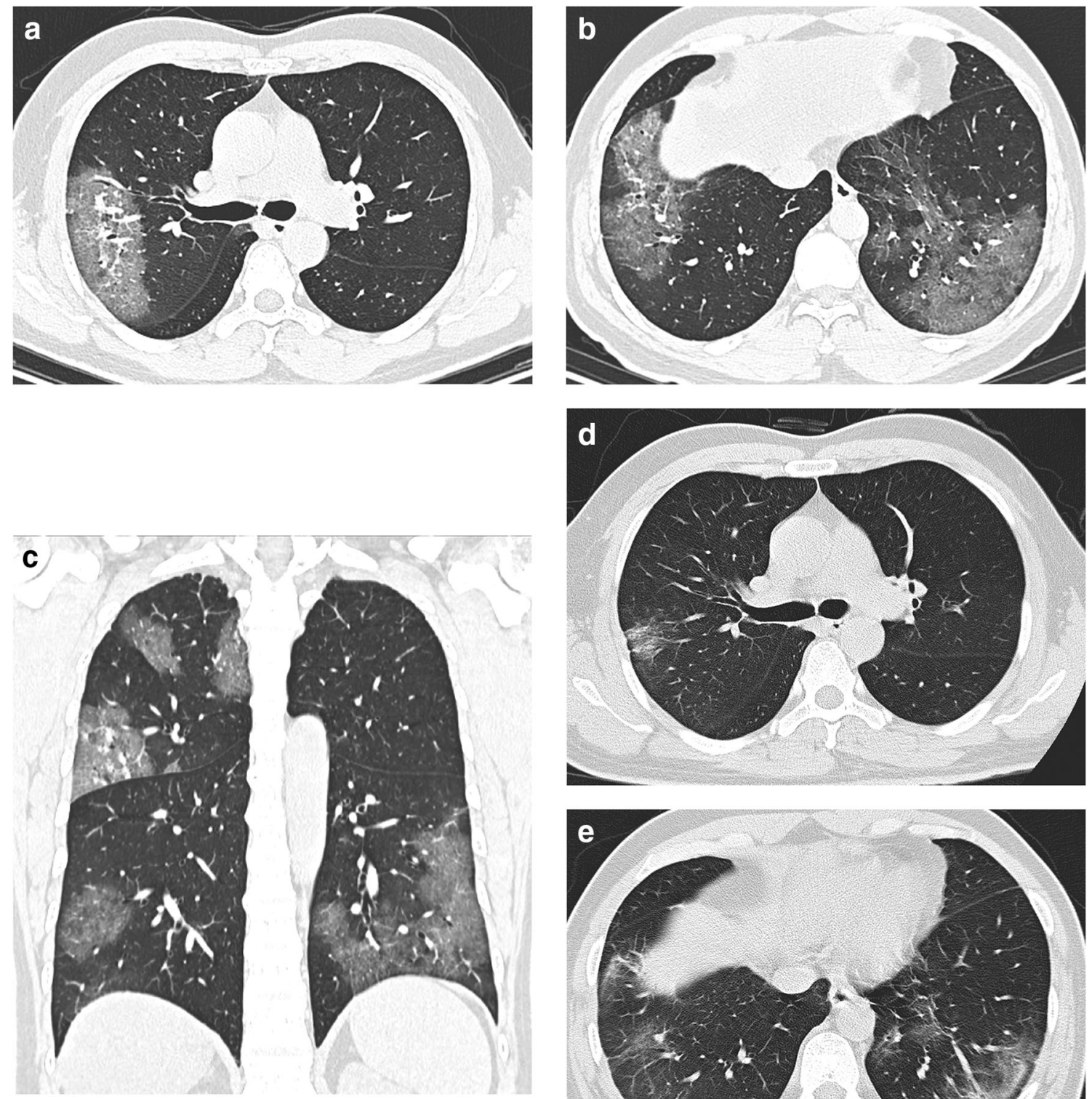

Fig. 5 A 44-year-old male was admitted to the hospital 1 day after fever and cough with a body temperature of $39^{\circ} \mathrm{C}$. The leukocytes were normal and lymphocytes were decreased. He was living in Zhuhai and traveled to Macao 12 days before the onset of the disease and stayed in Macao for 1 week. He was healthy and nonsmoker. Chest CT (images a-c) on the

was needed to find out the cause of clinical severity. Overall, we believe that many factors, such as advanced age, underlying diseases, and pleural effusions, would relate to the clinical severity, which call for a comprehensive evaluation.

\section{High proportion of mild-type patients}

Among the 78 cases, there were 24 cases of mild type $(30.8 \%)$ which mean those patients had positive real-
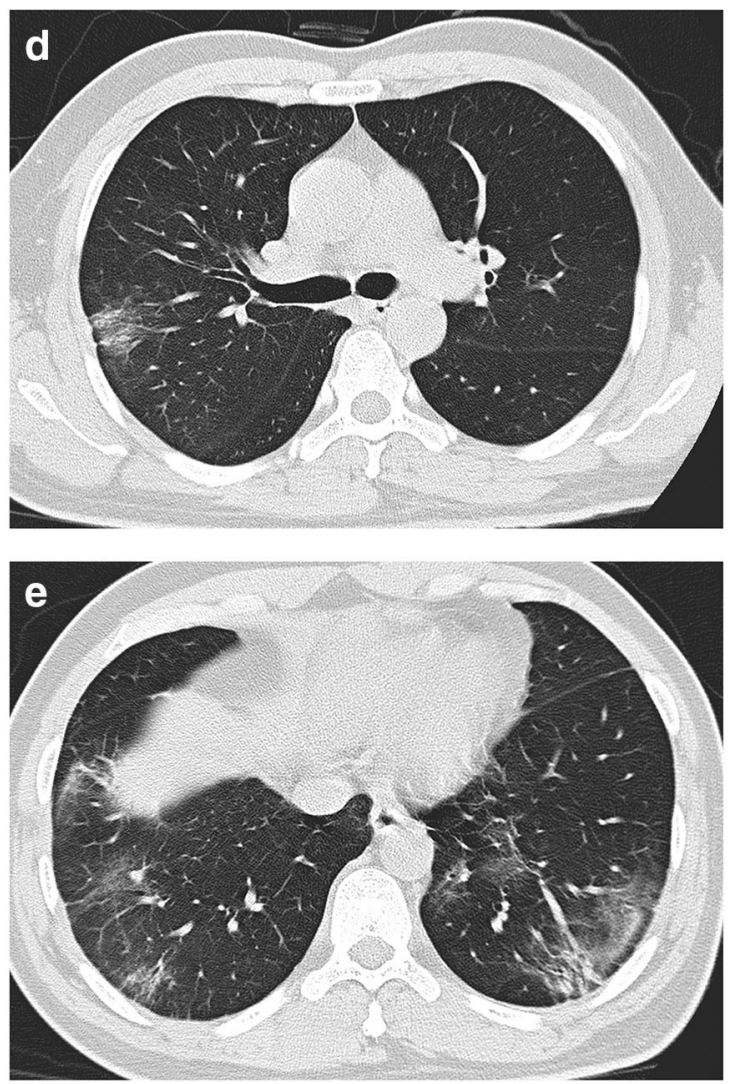

4th day after admission demonstrated bilateral peripheral ground-glass opacities without consolidation. TSS was 9. The clinical type was severe-critical type. Follow-up CT (images d, e) on the 22nd day after onset showed bilateral fibrotic changes with traction bronchiectasis and ground-grass opacities

time RT-PCR SARS-CoV-2 tests, while chest CT was normal. In the review of several recent published literature of COVID-19 in Wuhan, China, all patients reported in articles had ground-glass opacities in the lungs. Huang et al reported 41 infected cases in Wuhan, all had pneumonias [1]. Chen et al reported 99 confirmed cases in Wuhan with 74 bilateral pneumonias and 25 unilateral pneumonias [19]. However, the most recent report from Guangzhou had similar findings, which showed $23.6 \%$ 
confirmed patients without abnormalities on chest CT [20]. To further explore our data, we found several characteristics. Nine cases had a short time interval from onset to the latest CT examination with a range of 0-7 days, which indicated that the chest $\mathrm{CT}$ could be normal at the early phase. Another 9 cases had a longer time interval from onset to the latest CT scan with a range of 8-19 days. The negative findings may not relate to the shorter onset time. It remains to be further explored whether the CT negativity may relate to the degree of infection and autoimmunity. Finally, the last 6 patients had no symptoms. These patients were negative in both clinical and imaging, suggesting that some cases were potential sources of infection, which should be paid more attention to.

\section{Limitation}

In this study, the number of cases between groups was significantly different because too few severe-critical patients were included in this study, which decreased the reliability of statistical results. Only image analysis was carried out without combining clinical information in this study; however, advanced age, underlying diseases, and pleural effusions may lead to a lower TSS but severe situation. In our next study, we will include more cases, and make a comprehensive evaluation combining the clinical characteristics and laboratory examination information.

\section{Conclusion}

The proportion of clinical mild-type patients with COVID-19 was relatively high, screening for COVID19 with chest CT alone can lead to misdiagnosis in some patients, which would lead to a potential infection risk, so CT was not suitable as an independent screening tool. Visual quantitative analysis based on CT images has high consistency and high diagnostic ability, which can reflect clinical classification; it is expected to accurately assess the clinical severity of COVID-19 and guide the clinical treatment by combining with the clinical information.

Acknowledgments The authors thank Claudia Henschke, David Yankelevitz, and Rowena Yip from Mount Sinai Medical center for polishing the manuscript.

Funding information The authors state that this work has not received any funding.

\section{Compliance with ethical standards}

Guarantor The scientific guarantor of this publication is Shaolin Li.
Conflict of interest The authors of this manuscript declare no relationships with any companies, whose products or services may be related to the subject matter of the article.

Statistics and biometry No complex statistical methods were necessary for this paper.

Informed consent Written informed consent was waived by the Institutional Review Board.

Ethical approval Institutional Review Board approval was obtained.

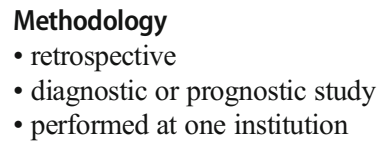

\section{References}

1. Huang C, Wang Y, Li X et al (2020) Clinical features of patients with 2019 novel coronavirus in Wuhan, China. Lancet. https://doi. org/10.1016/S0140-6736(20)30183-5

2. Lu R, Zhao X, Li J et al (2020) Genomic characterization and epidemiology of 2019 novel coronavirus: implications of virus origins and receptor binding. Lancet. https://doi.org/10.1016/S01406736(20)30251-8

3. Zhu N, Zhang D, Wang W et al (2020) A novel coronavirus from patients with pneumonia in China, 2019. N Engl J Med. https://doi. org/10.1056/NEJMoa2001017

4. WHO main website. https://www.who.int. Accessed March 12th, 2020

5. Phan LT, Nguyen TV, Luong QC et al (2020) Importation and human-to-human transmission of a novel coronavirus in Vietnam. N Engl J Med. https://doi.org/10.1056/NEJMc2001272

6. Rothe C, Schunk M, Sothmann P et al (2020) Transmission of 2019-nCoV infection from an asymptomatic contact in Germany. N Engl J Med. https://doi.org/10.1056/NEJMc2001468

7. Holshue ML, DeBolt C, Lindquist S et al (2020) First case of 2019 novel coronavirus in the United States. N Engl J Med. https://doi. org/10.1056/NEJMoa2001191

8. National Health Commission of the People's Republic of China. http://www.nhc.gov.cn. Assessed on March 12th, 2020

9. Chung M, Bernheim A, Mei X et al (2020) CT imaging features of 2019 novel coronavirus (2019-nCoV). Radiology:200230. https:// doi.org/10.1148/radiol.2020200230

10. Lei J, Li J, Li X, Qi X (2020) CT imaging of the 2019 novel coronavirus (2019-nCoV) pneumonia. Radiology. https://doi.org/ 10.1148/radiol.2020200236

11. Fang Y, Zhang H, Xu Y, Xie J, Pang P, Ji W (2020) CT Manifestations of two cases of 2019 novel coronavirus (2019nCoV) pneumonia. Radiology. https://doi.org/10.1148/radiol. 2020200280

12. Shi H, Han X, Zheng C (2020) Evolution of CT manifestations in a patient recovered from 2019 novel coronavirus (2019-nCoV) pneumonia in Wuhan, China. Radiology. https://doi.org/10.1148/radiol. 2020200269

13. Chinese Society of Radiology (2020) Imaging diagnosis of 2019nCoV pneumonia: expert recommendation of Chinese Society of Radiology, the first edition. Chin J Radiol 54(00):E001-E001. https://doi.org/10.3760/cma.j.issn.1005-1201.2020.0001

14. Hansell DM, Bankier AA, MacMahon H, McLoud TC, Muller NL, Remy J (2008) Fleischner society: glossary of terms for thoracic 
imaging. Radiology. 246(3):697-722. https://doi.org/10.1148/ radiol.2462070712

15. General Office of National Health Committee. Notice on the issuance of a program for the diagnosis and treatment of novel coronavirus (2019-nCoV) infected pneumonia (trial revised fifth edition). http://www.nhc.gov.cn/yzygj/s 7653 p/202003/ 46c9294a7dfe4cef80dc7f5912eb1989.shtml. Accessed 3 Mar 2020

16. Kim EA, Lee KS, Primack SL et al (2002) Viral pneumonias in adults: radiologic and pathologic findings. Radiographics $22 \mathrm{Spec}$ No:S137-S149. https://doi.org/10.1148/radiographics.22.suppl_1. g02oc15s 137

17. Koo HJ, Lim S, Choe J, Choi SH, Sung H, Do KH (2018) Radiographic and CT features of viral pneumonia. Radiographics. 38(3):719-739
18. Ooi GC, Khong PL, Müller NL et al (2004) Severe acute respiratory syndrome: temporal lung changes at thin-section $\mathrm{CT}$ in 30 patients. Radiology 230(3):836-844 75

19. Chen N, Zhou M, Dong X et al (2020) Epidemiological and clinical characteristics of 99 cases of 2019 novel coronavirus pneumonia in Wuhan, China: a descriptive study. Lancet. https://doi.org/10.1016/ S0140-6736(20)30211-7

20. Guan W, Ni Z, Hu Y et al (2020) Clinical characteristics of coronavirus disease 2019 in China. N Engl J Med. https://doi.org/10.1056/ NEJMoa2002032

Publisher's note Springer Nature remains neutral with regard to jurisdictional claims in published maps and institutional affiliations. 